\title{
ESTIMATIVA E MODELAGEM DOS ESTOQUES DE CARBONO EM SOLOS SOB ÁREAS DE CAMPO LIMPO ÚMIDO DO DISTRITO FEDERAL
}

\section{Estimates and modelling carbon stocks in soil under humid grassland areas in the Federal District of Brazil}

\author{
Andreia Maria da Silva França \\ Instituto Federal de Brasília, Brasília, Distrito Federal, Brasil \\ andreia.franca@ifb.edu.br \\ Rodrigo José de Oliveira Paiva \\ drigoj@gmail.com
}

Artigo recebido em 15/09/2014 e aceito para publicação em 02/02/2015

RESUMO: Este trabalho teve como principal objetivo a obtenção de parâmetros quantitativos básicos sobre os estoques de carbono em áreas de Campo Limpo Úmido, além da modelagem de função para a estimação do conteúdo de carbono orgânico do solo(COS) a partir de parâmetros físicos do solo (ex. textura, densidade). Foram selecionados 4 regiões com Campo Limpo Úmido no Distrito Federal, caracterizadas pelo baixo impacto antrópico, sendo 2 delas sobre gleissolo e 2 sobre plintossolo, tipos de solo mais comuns nessas regiões.. Em cada uma delas foi demarcado um transecto com 4 pontos equidistantes e realizada coleta de material em sete profundidades: 0-5, 5-10, 10-20, 20-30, 30-40, 40-50 e 50-60 cm. Foram determinadas a textura, a densidade aparente e o teor de carbono em cada profundidade. $\mathrm{O}$ estoque de carbono médio para as áreas de Campo Limpo Úmido até 60 $\mathrm{cm}$ de profundidade foi de $243.16 \mathrm{Mg} \mathrm{C} \mathrm{ha}^{-1} \mathrm{e}$ o estoque total estimado para essas áreas no Distrito Federal foi de $205.86 \mathrm{Gg}$ C. Os estoques de carbono e o COS em áreas degleissolo foram maiores do que em áreas de plintossolo. A função obtida com a modelagem das variáveis independentes (densidade, areia, argila e silte) mostrou-se capaz de estimar adequadamente o COS, tanto para gleissolo $\left(\mathrm{R}^{2}=0,88, \mathrm{RMSD}=1,48 ; \mathrm{RMSD}\right.$ validação $\left.=1,49\right)$ quanto para plintossolo $\left(\mathrm{R}^{2}=0,77\right.$, RMSD $=1,57 ;$ RMSD validação $=1,62$ ).

Palavras chave: Estoque de Carbono. Cerrado. Áreas Úmidas. Campo Limpo Úmido.

ABSTRACT: The main purpose of this study was to obtain basic quantitative parameters for carbon storage and modeling the soil organic carbon (SOC) in Humid Grassland (Campo Limpoúmido), a kind of grassland phytophysiognomy found in the Cerrado. We selected 4 regions of the Federal District with this kind of vegetation formation, characterized by low anthropic impact. In each one of the sampled regions, we marked a transect with 4 equidistant points and collected material at 7 different levels of depth: $0-5,5-10,10-20,20-30,30-40,40-50$ and 50-60 $\mathrm{cm}$. We determined the texture, bulk density and concentration of carbon at each depth. The average carbon storage for Humid 
Grassland areas, down to $60 \mathrm{~cm}$ deep, was $243.16 \mathrm{Mg} \mathrm{C} \mathrm{ha}^{-1}$ and the total estimated stock for these areas in the Federal District was $205.86 \mathrm{Gg} \mathrm{C}$. In general, the samples obtained in gleysols showed a carbon stockand SOC superior to those in plinthosols. The function obtained with the modeling of the independent variables (bulk density, sand, clay and silt) proved to be able to properly estimate the $\mathrm{SOC}$ in gleysol $\left(\mathrm{R}^{2}=0,88, \mathrm{RMSD}=1,48 ; \mathrm{RMSD}\right.$ validação $\left.=1,49\right)$ and in plinthosol $\left(\mathrm{R}^{2}=0,77\right.$, $\mathrm{RMSD}=1,57$; RMSD validação $=1,62$ ).

Keywords: Carbon Stocks. Cerrado. Wetland. Grasslands.

\section{INTRODUÇÃO}

$\mathrm{O}$ aumento da concentração de Gases de Efeito Estufa (GEE) - $\mathrm{CO}_{2}, \mathrm{~N}_{2} \mathrm{O}$ e o $\mathrm{CH}_{4}$ - na atmosfera terrestre e sua estreita relação com as mudanças climáticas torna de grande importância o estudo desses elementos no meio ambiente. Diferentes compartimentos do ciclo biogeoquímico do carbono estão envolvidos na liberação de compostos de carbono para a atmosfera, sendo de particular relevância o carbono presente no compartimento solo. Esse representa cerca de quatro vezes mais do que o presente na biomassa vegetal e quase três vezes mais do que o presente na atmosfera(LAL; FOLLETT, 2009). Ambientes como formações vegetacionais de tipo campestre e áreas úmidas podem exercer um papel especialmente importante na mitigação desse processo, em especial pelas condições ambientais específicas sob as quais são mantidas que favorecem a acumulação de carbono orgânico.

Áreas campestres são responsáveis pela cobertura de grandes extensões de hábitat natural no mundo (SCURLOCK; HALL, 1998). Esse tipo de formação, caracterizada pelo predomínio de um estrato herbáceo contínuo e a ausência de árvores ou arbustos, têm até $60 \%$ de toda sua ocorrência na região tropical, especialmente em ecossistemas de savana (SCURLOCK; HALL, 1998). No Brasil, esse tipo de ambiente está representado principalmente no Bioma Cerrado.Grace et al. (2006) indicaram que entre $10 \% \mathrm{e}$ $30 \%$ de todo o carbono presente nos solos, localizam-se em regiões de savana tropical.

Os solos sob áreas úmidas, por sua vez, são reconhecidos na literatura como importantes áreas estocadoras e fontes potenciais para a liberação de carbono, devido, principalmente, às condições anaeróbicas sob as quais são mantidas (baixo nível de oxigênio, alta quantidade de água, baixa difusividade de gases). Tais condições favorecem o acúmulo de matéria orgânica e carbono no solo (COLETTI et al., 2013). Pequenas diferenças climáticas, hidrológicas e no uso da terra podem alterar de forma importante o delicado balanço dos estoques de carbono dessas regiões, provocando sua liberação para a atmosfera (NEUE et al., 1997).

Ainda que o predomínio das fitofisionomias no Cerrado sejam as associadas a solos bem drenados, com formações savânicas, em que estão presentes arbustos, ocorrem nesse bioma diferentes fitofisionomias sobre solos periodicamente inundados, correspondendo a ecossistemas de áreas úmidas(WALTER; CARVALHO; RIBEIRO, 2008). O Campo Limpo Úmido é uma delas. Esse tipo de ambiente tem sua ocorrência onde o lençol freático é superficial, especialmente em áreas de nascentes, em encostas, nos fundos dos vales e bordeando as matas de galeria, em solos hidromórficos(WALTER; CARVALHO; RIBEIRO, 2008). Caracteriza-se pelo predomínio de um estrato herbáceo contínuo, ausência de árvores ou arbustos, além de grande abundância e riqueza de espécies da família Poaceae (MUNHOZ; FELFILI, 2008).

Apesar de sua potencial importância para a preservação do carbono no solo, até o momento, poucos estudos abordaram áreas de Campo Limpo Úmido(FERREIRA et al., 2008; MEIRELLES; FERREIRA; FRANCO, 2006).Trabalhos envolvendo a modelagem e desenvolvimento de funções de pedotransferênciapara solos do Cerrado têm observado uma relação entre o conteúdo de carbono orgânico no solo (COS) e suas características físicas e estruturais, como a textura, densidade e mineralogia (FRANÇA, 2011; REIN; DUXBURY, 2008; ZINN et al., 2007).O desenvolvimento de tais funções permite a estimação dos estoques de carbono a partir de informações com menor custo e maior rapidez para sua obtenção. 
Considerando a potencial importância de fitofisionomias de Campo Limpo Úmido para a manutenção do carbono orgânico no solo, evitando sua emissão para a atmosfera, e com a finalidade de fornecer meios menos dispendiosos para a estimação dos estoques de carbono nesse tipo de ambiente, temos porprincipal objetivo neste estudoa obtenção de parâmetros quantitativos básicos dos solos nas regiões de ocorrência de Campos Limpos Úmidos, bem como o desenvolvimento de função que permita a estimação do carbono orgânico.

\section{MATERIAIS E MÉTODOS}

\section{Area de Estudo}

O estudo foi realizado no Distrito Federal (Brasil), o qual compreende uma área de $5.779 \mathrm{~km}^{2}$, localizado na região central do Bioma Cerrado (Figura
1). Essa região é caracterizada como uma região típica de Cerrado, composta por um mosaico heterogêneo de diferentes formações vegetacionais (EITEN, 1972; WALTER; CARVALHO; RIBEIRO, 2008), abrangendo cerca de $0,3 \%$ da área total do Cerrado (2,03 milhões de $\mathrm{km}^{2}$ ).A maior parte da paisagem natural na região é caracterizada por planaltos com solos bem drenados, mas também há a ocorrência de regiões periodicamente úmidas ou alagadas (SILVA et al., 2006).O clima na região é tropical úmido (Aw - classificação de Köppen), caracterizado por marcada sazonalidade, com invernos secos e verões chuvosos. Cerca de 70\%do Distrito Federalé ocupado por regiões com classes de uso antrópico (IBAMA, 2014), sendo a maior parte das áreas naturais bem preservadas situadas em propriedades pública destinadas à conservação do ambiente natural ou à pesquisa, a exemplo de unidades de conservação e unidades dedicadas à pesquisa ambiental.

Figura 1 - Mapa dos Campos Limpos Úmidos do Distrito Federal e áreas amostradas.

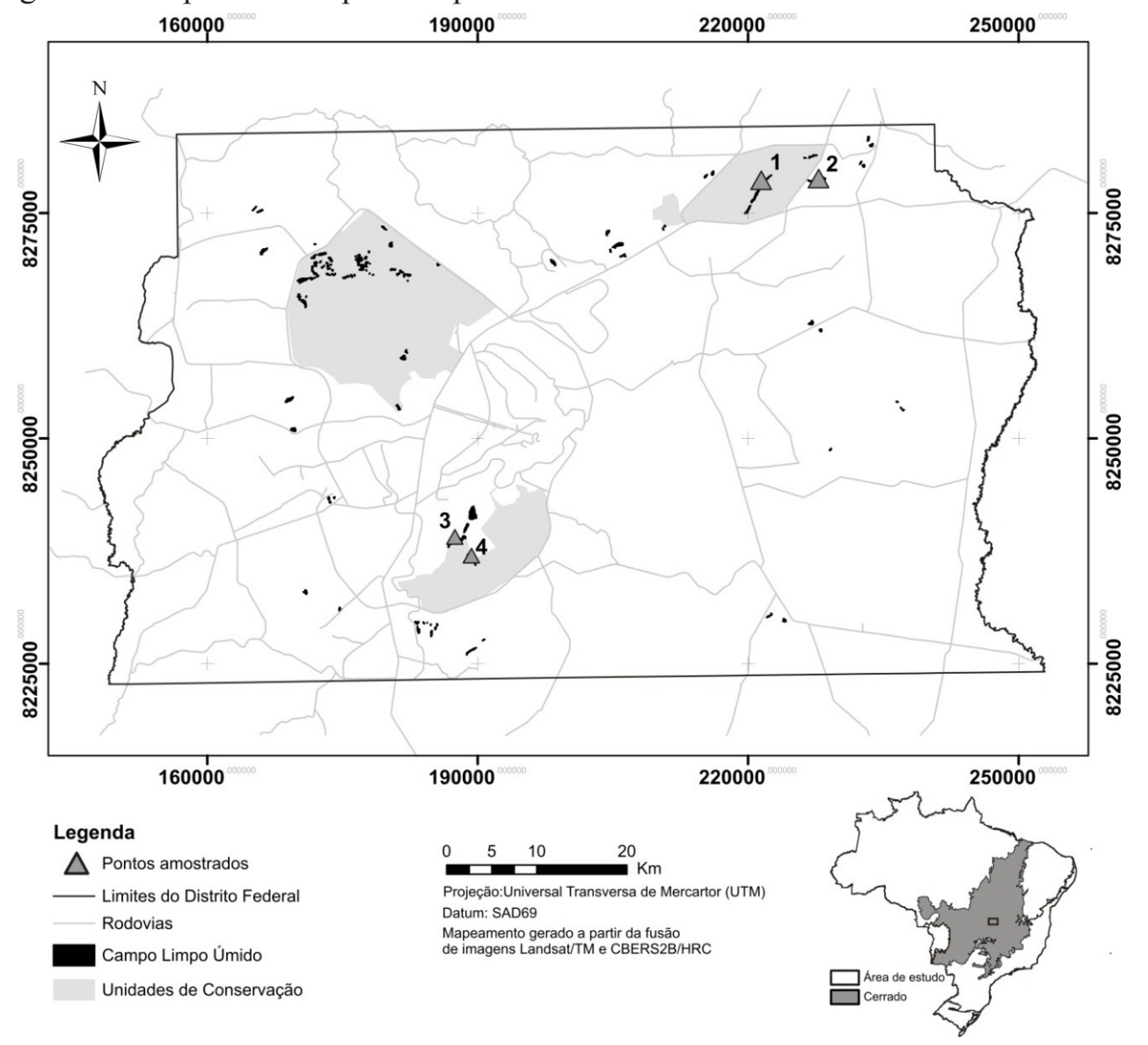

Fonte: elaboração dos autores. 
As áreas de Campo Limpo Úmido normalmente situam-se em faixas horizontais sobre lados de vales, separando o Cerrado Stricto Senso da Mata de Galeria. Na maioria das vezes, essas áreas formam limites bem definidos com essas fitofisionomias, numa região com abrupta transição entre diferentes comunidades biológicas e grande diversidade. Por serem áreas úmidas, nessas regiões o lençol freático tende a ser raso e frequentemente aflora à superfície. Os solos permanecem grande parte do ano saturado de água, impedindo ou dificultando sua coleta em períodos em que o lençol freático se encontra próximo à superfície.Os tipos mais comuns de solo nesse tipo de fitofisionomiasão o gleissolo e o plintossolo.

O mapeamento de áreas de Campo Limpo Úmido no Distrito Federal foi realizado por França \& Sano (2011), com a utilização de imagens multiespectrais - Landsat5/TM e CBERS2B/HRC e o uso de técnicas de fusão IHS. Como resultado, houve a identificação de 846,61 ha de Campo Limpo Úmido no Distrito Federal (0,14\% de toda a área de estudo).

\section{Características do solo}

Os solos presentes nas áreas estudadas são os gleissolos e os plintossolos. Os gleissolos formamse a partir de sedimentos aluviais, com presença de lençol freático próximo à superfície na maior parte do ano, caracterizando um ambiente de acúmulo de matéria orgânica e de oxirredução (REATTO et al., 1998). São solos que ocasionalmente podem ter textura arenosa (areia ou areia franca) somente nos horizontes superficiais, desde que seguidos de horizonte glei de textura franco arenosa ou mais fina (EMBRAPA, 2006; FAO, 2006). São solos desenvolvidos em materiais inconsolidados (sedimentos ou saprolito) e muito influenciados por ocorrências de encharcamento prolongado. Tais condições são normalmente ocasionadas por um lençol freático próximo à superfície, pelo menos em alguns meses do ano, o que deixa os poros saturados com água por tempo relativamente prolongado. Essa saturação, na presença de matéria orgânica, diminui o oxigênio dissolvido e provoca a redução química e dissolução dos óxidos de ferro, que é transformado, e parcialmente removido, o que faz com que surjam cores acinzentadas no horizonte subsuperficial (LEPSCH, 2002).

Os plintossolos correspondem a solos minerais hidromórficos, com séria restrição à percolação de água, encontrados em situações de alagamento temporário e, portanto, escoamento lento (REATTO et al., 1998). Morfologicamente apresentam horizonte de subsuperfície com manchas avermelhadas distribuídas no perfil, de aspecto variegado (resultado da concentração de ferro do solo), chamadas de plintita (EMBRAPA, 2006; FAO, 2006). São solos formados sob condições de algum impedimento ao movimento da água gravitativa, mormente em locais em que há grande oscilação do lençol freático facilitando a formação da plintita (LEPSCH, 2002). Ocorrem em regiões quentes e úmidas, especialmente naquelas com estação seca bem definida ou período com marcante diminuição da precipitação pluvial (OLIVEIRA; JACOMINE; CAMARGO, 1992).

\section{Amostragem de campo}

No Distrito Federal foram selecionados quatro sítios de amostragem com características típicas de Campo Limpo Úmido. Dois deles situaram-se na Estação Ecológica Águas Emendadas (área 1 - gleissolo e área 2 - plintossolo) e dois deles na Fazenda Água Limpa (área 3 - gleissolo e área 4 - plintossolo), unidade de pesquisa de propriedade da Universidade de Brasília. As áreas amostradas foram escolhidas por manterem preservadas suas características naturais, apresentando baixo impacto antrópico. A amostragem foi realizada durante $o$ período de seca.

Em cada um dos quatro Campos Limpos Úmidos selecionados, foi delimitado um transecto na região central, iniciando na borda do Cerrado sensu stricto e finalizando na Mata de Galeria, atravessando toda a sua extensão. Em cadatransecto foram demarcados 4 pontos amostraisequidistantes onde foram coletadas amostras em sete diferentes profundidades: $0-5 \mathrm{~cm}, 5-10 \mathrm{~cm}, 10-20 \mathrm{~cm}, 20-30$ $\mathrm{cm}, 30-40 \mathrm{~cm}, 40-50 \mathrm{~cm}$ e $50-60 \mathrm{~cm}$. 
Para a determinação do teor de carbono e textura do solo foram coletadas amostras deformadas utilizando trado holandês e para a determinação da densidade aparente do solo foram coletadas amostras indeformadas com auxílio de anéis volumétricos. Como resultado, obteve-se112 amostras, sendo 56 amostras referentes às áreas de Campo Limpo Úmido sob gleissoloe 56 amostras referentes às áreas de Campo Limpo Úmido sob plintossolo.

\section{Preparação das amostras e análises laboratoriais}

Em relação a características texturais do solo, foi determinadoo conteúdo de areia, argila e siltepara todos os intervalos de profundidade por meio de análise granulométrica das amostras deformadas, distinguindo suas frações em classes de acordo com seus diâmetros: argila $(0,000-0.002 \mathrm{~mm})$, silte $(0.002-0.063 \mathrm{~mm})$ e areia $(0.063-2 \mathrm{~mm})$.

A densidade aparente foi determinada obtendo-se amostras indeformadas de solo em diferentes profundidades, com a utilização de anel de aço de bordos cortantes e capacidade interna de $100 \mathrm{~cm}^{3}$. As amostras obtidas foram secas em estufa a $110{ }^{\circ} \mathrm{C}$ e pesadas com utilização de instrumento de precisão. A densidade aparente $(\mathrm{Da})$ foi determinada como função da massa de solo seco e do volume conhecido ( $\mathrm{Da}=$ Massa/Volume). Nenhuma das amostras obtidas para a análise da densidade conteve quantidade significativa de rochas ou fragmentos, não sendo necessária a correção dos valores obtidos.

As amostras de solo coletadas para a determinação do teor de carbono foram secas ao ar e posteriormente destorroadas e passadas em peneira de $2 \mathrm{~mm}$. Essas amostras foram trituradas com o auxílio de um moedor e utilizando-se gral e pistilo até que as partículas pudessem passar por peneira de malha de $0,25 \mathrm{~mm}$. A cada troca de conjunto de amostras foi realizada a higienização de todo o material (moedor, gral, pistilo e peneiras), a fim de se evitar sua contaminação. Subamostras foram pesadas entre 20 e $30 \mathrm{mg}$, com precisão de 4 casas decimais, em cápsulas de estanho e submetidas à análise para determinação do teor de carbono total no solo (TOC).

O teor de carbono nas amostras de solo foi determinado por meio do método analítico por combustão seca a $900^{\circ} \mathrm{C}(\mathrm{CHNS} / \mathrm{O})$ em analisador elementar (Perkin Elmer, PE-2400 Séries II). Nesse método, uma pequena amostra de solo é aquecida até $900^{\circ} \mathrm{C}$ e é medido o $\mathrm{CO}^{2}$ resultante da combustão do material. Os resultados são expressos como percentual e refere-se ao carbono orgânico e inorgânico presente nas amostras (TIESSEN; MOIR, 1993). Foi realizado um total de 112 medições e a cada 10 amostras o aparelho foi calibrado com amostras-padrão cistina.

\section{Modelagem e validação da função de pedotransferência}

A função para a estimação do $\operatorname{COS}$ foi obtida com a utilização do algoritmo de optimização deLevenberg-Marquardt (LEVENBERG, 1944; MARQUARDT, 1963)por meio dosoftware Matlab 2013(MATLAB, 2013). O algoritmo mencionado tem por objetivo encontrar o melhor ajuste entre uma função objetivo e os dados observados, promovendo a redução da distância entre valores estimados e observados (redução de mínimos quadrados). Para a definição da função objetivo, a exemplo de Zinnet al. (2012), regressões lineares foram empregadas para detectar possíveis relações entre teores de $\operatorname{COS}$ e as variáveisindependentes consideradas (profundidade, densidade, areia, argila e silte). Além dos dados originais, foram submetidas à regressão transformações logarítmicas das variáveis e a soma ou multiplicação entre até duas variáveis dependentes. Os termos integrantes da função objetivo foram definidos como aqueles que apresentassem as melhores características para a regressão linear (maior R $\mathrm{R}^{2}$ e menor Root Mean Square Deviation RMSD).

Para a seleção dos coeficientes, a função objetivo foi submetida a500 interações realizadas com diferentes conjuntos de dados, sendo tomados randomicamente 46 registros para treinamento e 10 registros para validação. Com o procedimento mencionado foi possível se obter os coeficientes (valores médios) e o ajuste médio dos valores estimados de COS aos dados observados, bem como aos dados de validação (SEE, $\mathrm{R}^{2}, \mathrm{R}^{2}$ ajustado e RMSD). 


\section{Cálculos do Estoque de Carbono no Solo}

O estoque de carbono orgânico no solo foi quantificado em base volumétrica expressa em $\mathrm{Mg}$ $\mathrm{C}$ ha $^{-1}$ para as camadas $0-5 \mathrm{~cm}, 5-10 \mathrm{~cm}, 10-20$ $\mathrm{cm}, 20-30 \mathrm{~cm}, 30-40 \mathrm{~cm}, 40-50 \mathrm{~cm}$ e $50-60 \mathrm{~cm}$ de profundidade. $\mathrm{O}$ cálculo do estoque de carbono para uma determinada profundidade $(\rho, \mathrm{cm})$ foi realizado com o uso da função:

$$
\begin{gathered}
\mathrm{EC}=\mathrm{C} \cdot \rho \cdot \mathrm{e} / 10 \\
\text { equação }(1)
\end{gathered}
$$

Onde:

$\mathrm{EC}=$ estoque de $\mathrm{C}$ orgânico em determinada profundidade $\left(\mathrm{Mg} \mathrm{ha}^{-1}\right)$

$\mathrm{C}=$ teor de $\mathrm{C}$ orgânico total na profundidade amostrada $\left(\mathrm{g} \mathrm{kg}^{-1}\right)$ $\rho=$ densidade aparente do solo da profundidade $\left(\mathrm{kg} \mathrm{dm}^{-3}\right)$

$$
\mathrm{e}=\text { espessura da camada considerada }(\mathrm{cm})
$$

\section{RESULTADOS}

Para ambos os tipos de solos amostrados, gleissolose plintossolos, foi observada textura predominantemente argilo-siltosa, com um menor conteúdo de areia ao longo do perfil (Tabela 1).

Tabela 1 - características texturais e físicas de gleissolos e plintossolosem áreas de Campo Limpo Úmido(média \pm erro padrão).

\begin{tabular}{llllll}
\hline $\begin{array}{l}\text { Solo / } \\
\text { Profundidade }\end{array}$ & $\mathbf{N}$ & $\begin{array}{l}\text { Densidade } \\
(\mathbf{k g ~ d m - 3})\end{array}$ & $\begin{array}{l}\text { Areia } \\
\mathbf{( \% )}\end{array}$ & $\begin{array}{l}\text { Argila } \\
\mathbf{( \% )}\end{array}$ & $\begin{array}{l}\text { Silte } \\
\mathbf{( \% )}\end{array}$ \\
\hline Gleissolo & $\mathbf{5 6}$ & $\mathbf{0 . 5 1} \pm \mathbf{0 . 0 4}$ & $\mathbf{9 . 1 6} \pm \mathbf{0 . 7 7}$ & $\mathbf{4 4 . 7 7} \pm \mathbf{2 . 3 6}$ & $\mathbf{4 6 . 0 7} \pm \mathbf{2 . 6 1}$ \\
$0-10 \mathrm{~cm}$ & 16 & $0.34 \pm 0.04$ & $10.63 \pm 1.81$ & $44.88 \pm 3.82$ & $44.5 \pm 4.45$ \\
$0-5 \mathrm{~cm}$ & 8 & $0.32 \pm 0.06$ & $11.50 \pm 2.78$ & $47.25 \pm 5.02$ & $41.25 \pm 5.89$ \\
$5-10 \mathrm{~cm}$ & 8 & $0.35 \pm 0.06$ & $9.75 \pm 2.48$ & $42.50 \pm 5.97$ & $47.75 \pm 6.88$ \\
\hline $10-20 \mathrm{~cm}$ & 8 & $0.43 \pm 0.07$ & $11.50 \pm 2.24$ & $37.75 \pm 5.47$ & $50.75 \pm 6.28$ \\
\hline $20-30 \mathrm{~cm}$ & 8 & $0.51 \pm 0.09$ & $8.75 \pm 2.37$ & $43.88 \pm 6.67$ & $47.38 \pm 7.82$ \\
\hline $30-40 \mathrm{~cm}$ & 8 & $0.56 \pm 0.10$ & $7.50 \pm 1.40$ & $45.38 \pm 6.81$ & $47.13 \pm 7.50$ \\
\hline $40-50 \mathrm{~cm}$ & 8 & $0.66 \pm 0.11$ & $7.25 \pm 0.92$ & $47.50 \pm 6.99$ & $45.25 \pm 7.60$ \\
\hline $50-60 \mathrm{~cm}$ & 8 & $0.76 \pm 0.11$ & $7.88 \pm 1.59$ & $49.13 \pm 7.93$ & $43.00 \pm 8.32$ \\
\hline Plintossolo & $\mathbf{5 6}$ & $\mathbf{0 . 6 6} \pm \mathbf{0 . 0 4}$ & $\mathbf{1 2 . 1 4} \pm \mathbf{0 . 6 5}$ & $\mathbf{4 6 . 2 1} \pm \mathbf{1 . 7 1}$ & $\mathbf{4 1 . 6 4} \pm \mathbf{1 . 9 5}$ \\
\hline $0-10 \mathrm{~cm}$ & 16 & $0.41 \pm 0.04$ & $8.5 \pm 0.77$ & $37.44 \pm 2.36$ & $54.06 \pm 2.92$ \\
\hline $0-5 \mathrm{~cm}$ & 8 & $0.37 \pm 0.05$ & $8.00 \pm 1.09$ & $37.13 \pm 3.62$ & $54.88 \pm 4.34$ \\
$5-10 \mathrm{~cm}$ & 8 & $0.45 \pm 0.05$ & $9.00 \pm 1.15$ & $37.75 \pm 3.28$ & $53.25 \pm 4.20$ \\
\hline $10-20 \mathrm{~cm}$ & 8 & $0.56 \pm 0.06$ & $13.25 \pm 1.03$ & $38.63 \pm 3.41$ & $48.13 \pm 3.50$ \\
\hline $20-30 \mathrm{~cm}$ & 8 & $0.67 \pm 0.08$ & $12.50 \pm 1.13$ & $44.13 \pm 4.46$ & $43.38 \pm 5.12$ \\
\hline $30-40 \mathrm{~cm}$ & 8 & $0.76 \pm 0.10$ & $15.50 \pm 2.20$ & $50.00 \pm 4.95$ & $34.50 \pm 4.45$ \\
\hline $40-50 \mathrm{~cm}$ & 8 & $0.90 \pm 0.08$ & $13.00 \pm 1.71$ & $56.50 \pm 2.12$ & $30.50 \pm 2.38$ \\
\hline $50-60 \mathrm{~cm}$ & 8 & $0.90 \pm 0.08$ & $13.75 \pm 2.11$ & $59.38 \pm 2.11$ & $26.88 \pm 1.48$ \\
\hline Campo Limpo Úmido & $\mathbf{1 1 2}$ & $0.59 \pm 0.03$ & $10.65 \pm 0.52$ & $45.49 \pm 1.45$ & $43.86 \pm 1.64$ \\
\hline
\end{tabular}

Em relação à textura, a principal diferença observada entre os tipos de solos foi um maior percentual 
de areia nas porções mais profundas do perfil (30$60 \mathrm{~cm}$ ) em plintossolos e uma maior quantidade de argila em gleissolosnessa mesma porção (Figura 2). Não houve diferença relevante entre os solos em relação ao conteúdo de silte, sendo observado um decréscimo de seu conteúdo com a profundidade para ambos os solos. A densidade do solo foi crescente ao longo do perfil $(0-60 \mathrm{~cm})$ e apresentou maiores valores observados para plintossolos. As maiores diferenças para a densidade também ocorreram para maiores profundidades $(30-60 \mathrm{~cm})$.

Figura2 - conteúdo de areia (a); argila (b); silte (c); e densidade aparente do solo (d) ao longo do perfil (0$60 \mathrm{~cm}$ ) em áreas de Campo Limpo Úmido sobre gleissolo e plintossolo.

a)

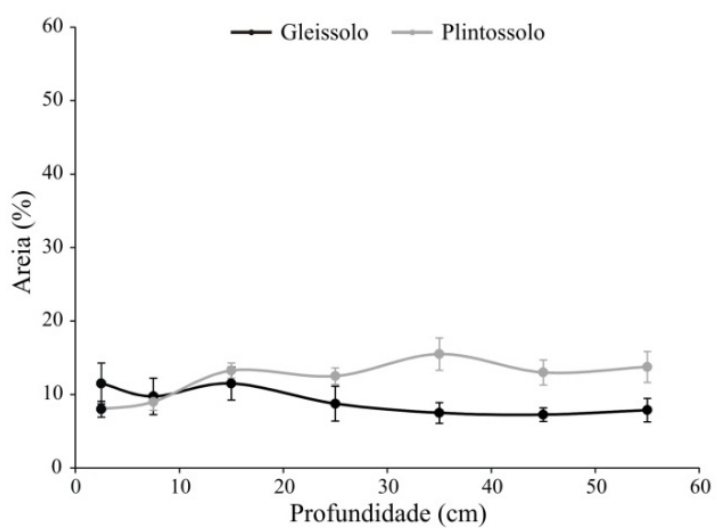

c)

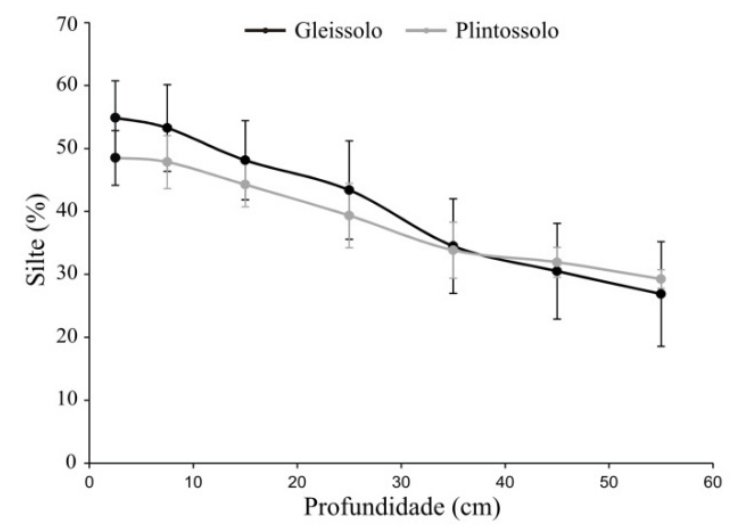

b)

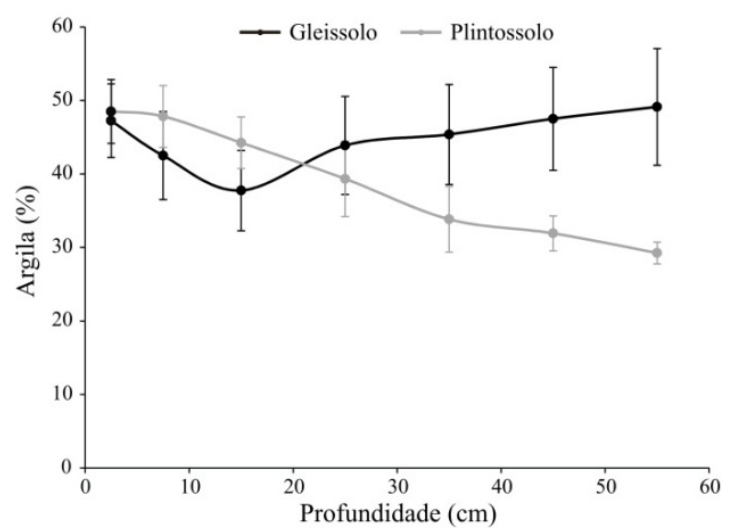

d)

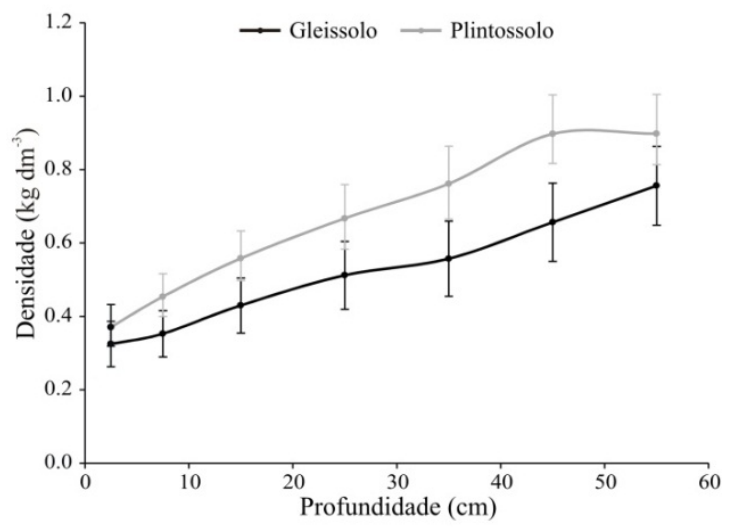

Fonte: elaboração dos autores.

A concentração de COS foi decrescente com a profundidade, sendo que plintossolos apresentaram menores valores ao longo de todo o perfil quando comparados a gleissolos (Tabela 2). O estoque de carbono também apresentou valores superiores em áreas de gleissolo (Tabela 2). 
Tabela 2-Valores observados (Obs.) e estimados (Est.) pelas funções obtidas para aconcentração de carbono orgânico no solo (COS) e estoque de carbono no solo em áreas de Campo Limpo Úmido (média \pm erro

\begin{tabular}{|c|c|c|c|c|c|}
\hline $\begin{array}{l}\text { Solo / } \\
\text { Profundidade }\end{array}$ & & $\begin{array}{l}\text { COS Obs. } \\
\left(\mathrm{g} \mathrm{kg}^{-1}\right)\end{array}$ & $\begin{array}{l}\text { COS Est. } \\
\left(\mathrm{g} \mathrm{kg}^{-1}\right)\end{array}$ & $\begin{array}{l}\text { Estoque Obs. } \\
\left(\mathrm{Mg} \mathrm{C} \mathrm{ha}^{-1}\right)\end{array}$ & $\begin{array}{l}\text { Estoque Est. } \\
(\mathrm{Mg} \mathrm{C} \mathrm{ha-1)}\end{array}$ \\
\hline Gleissolo & 60 & $84.16 \pm 5.68$ & $83.75 \pm 5.35$ & $258.84 \pm 36.32$ & $257.58 \pm 35.21$ \\
\hline $0-10 \mathrm{~cm}$ & 10 & $111.83 \pm 9.44$ & $113.83 \pm 6.59$ & $37.87 \pm 7.99$ & $38.55 \pm 7.11$ \\
\hline $0-5 \mathrm{~cm}$ & 5 & $116.14 \pm 14.79$ & $118.36 \pm 9.30$ & $18.85 \pm 6.00$ & $19.21 \pm 5.18$ \\
\hline $5-10 \mathrm{~cm}$ & 5 & $107.53 \pm 12.58$ & $109.29 \pm 9.68$ & $18.96 \pm 5.60$ & $19.28 \pm 5.15$ \\
\hline $10-20 \mathrm{~cm}$ & 10 & $95.06 \pm 12.58$ & $94.36 \pm 10.22$ & $40.85 \pm 12.52$ & $40.55 \pm 11.46$ \\
\hline $20-30 \mathrm{~cm}$ & 10 & $81.10 \pm 13.01$ & $81.06 \pm 12.20$ & $41.51 \pm 14.13$ & $41.49 \pm 13.71$ \\
\hline $30-40 \mathrm{~cm}$ & 10 & $76.66 \pm 14.99$ & $74.28 \pm 13.78$ & $42.71 \pm 16.20$ & $41.38 \pm 15.28$ \\
\hline $40-50 \mathrm{~cm}$ & 10 & $62.85 \pm 13.76$ & $60.75 \pm 14.01$ & $41.25 \pm 15.73$ & $39.87 \pm 15.67$ \\
\hline $50-60 \mathrm{~cm}$ & 10 & $49.78 \pm 13.15$ & $48.14 \pm 14.29$ & $37.62 \pm 15.28$ & $36.39 \pm 15.97$ \\
\hline Plintossolo & 60 & $54.35 \pm 4.80$ & $52.40 \pm 4.01$ & $214.49 \pm 31.05$ & $206.80 \pm 27.51$ \\
\hline $0-10 \mathrm{~cm}$ & 10 & $86.76 \pm 9.90$ & $82.69 \pm 5.36$ & $35.74 \pm 7.33$ & $34.06 \pm 5.31$ \\
\hline $0-5 \mathrm{~cm}$ & 5 & $92.90 \pm 16.31$ & $88.42 \pm 7.94$ & $17.20 \pm 5.41$ & $16.37 \pm 3.74$ \\
\hline $5-10 \mathrm{~cm}$ & 5 & $80.61 \pm 11.96$ & $76.96 \pm 7.13$ & $18.28 \pm 4.87$ & $17.45 \pm 3.68$ \\
\hline $10-20 \mathrm{~cm}$ & 10 & $64.36 \pm 9.88$ & $62.57 \pm 6.40$ & $35.90 \pm 9.38$ & $34.90 \pm 7.33$ \\
\hline $20-30 \mathrm{~cm}$ & 10 & $50.55 \pm 9.23$ & $51.50 \pm 8.93$ & $33.71 \pm 10.37$ & $34.34 \pm 10.25$ \\
\hline $30-40 \mathrm{~cm}$ & 10 & $39.28 \pm 7.76$ & $38.18 \pm 8.22$ & $29.90 \pm 9.69$ & $29.07 \pm 9.94$ \\
\hline $40-50 \mathrm{~cm}$ & 10 & $29.21 \pm 4.58$ & $26.39 \pm 5.23$ & $26.20 \pm 6.46$ & $23.68 \pm 6.81$ \\
\hline $50-60 \mathrm{~cm}$ & 10 & $23.53 \pm 2.57$ & $22.77 \pm 4.17$ & $21.11 \pm 4.28$ & $20.44 \pm 5.65$ \\
\hline Campo Limpo Úmido & 60 & $69.25 \pm 3.96$ & $68.07 \pm 3.65$ & $243.16 \pm 25.18$ & $239.02 \pm 23.87$ \\
\hline
\end{tabular}

O estoque de carbono médio observado para Campo Limpo Úmido foi de $243.16 \pm 25.17 \mathrm{Mg}$ $\mathrm{Cha}^{-1}$. Na porção do solo de 0-60 $\mathrm{cm}$, nos 846,61 hectaresocupados por áreas de Campo Limpo Úmido no Distrito Federal, estimou-se um estoque total de $205.86 \pm 21.30 \mathrm{Gg}$ C.

Observou-se uma maior relação na variação do COS com a densidade $\left(\mathrm{R}^{2}=0,75 ; \mathrm{p}<0,00\right)$, o produto entre a densidade e o logaritmo natural da profundidade $\left(\mathrm{R}^{2}=0.66 ; \mathrm{p}<0,00\right)$ e com o somatório entre areia e silte $\left(R^{2}=0.37 ; p=0,05\right)$. Respeitando a relação não-linear apresentada entre o conteúdo de carbono no solo e as variáveis independentes (densidade, profundidade, areia e silte), os melhores ajustes na relação entre o $\operatorname{COS}$ medido e o COS estimado foram obtidos com a função polinomial:

$$
\begin{gathered}
\mathrm{C}(\text { est })=\mathrm{X}_{1}+\mathrm{X}_{2}^{*}(\text { Dens. })^{\mathrm{X}}+ \\
\mathrm{X}_{4} * \ln \text { (Prof.) } *(\text { Dens. })+\mathrm{X}_{5}^{*}(\text { Areia })_{6}{ }_{6}+\mathrm{X}_{7}(\text { Silte })^{\mathrm{X}}{ }_{8} \\
\text { equação (2) }
\end{gathered}
$$


Onde:

C (est) - carbono orgânico estimado $\left(\mathrm{g} \mathrm{kg}^{-1}\right)$;

Dens. - densidade do solo $\left(\mathrm{kg} \mathrm{dm}^{-3}\right)$;

Prof. - profundidade - valores de entrada: $2,5 \mathrm{~cm} ; 7,5$ $\mathrm{cm} ; 15 \mathrm{~cm} ; 25 \mathrm{~cm} ; 35 \mathrm{~cm} ; 45 \mathrm{~cm} ; 55 \mathrm{~cm}$, referentes às classes de profundidade $0-5 \mathrm{~cm}, 5-10 \mathrm{~cm}, 10-20$ $\mathrm{cm}, 20-30 \mathrm{~cm}, 30-40 \mathrm{~cm}, 40-50 \mathrm{~cm}$ e $50-60 \mathrm{~cm}$; Areia - conteúdo de areia (\%);

Silte - conteúdo de silte (\%); $\mathrm{X}_{\mathrm{n}}$ - coeficientes; foram:

Os coeficientes determinados para as funções

Gleissolo: $\mathrm{X}_{1}=-3.2617 ; \mathrm{X}_{2}=6.6697 ; \mathrm{X}_{3}=-0.4543$; $X_{4}=-1.1727 ; X_{5}=-17.8626 ; X_{6}=0.0102 ; X_{7}=18.7415$; $\mathrm{X}_{8}=0.0412$;
Plintossolo: $X_{1}=-1.2428 ; X_{2}=2.6539 ; X_{3}=-0.6836$; $\mathrm{X}_{4}=-0.4019 ; \mathrm{X}_{5}=-44.4943 ; \mathrm{X}_{6}=-4.1142 ; \mathrm{X}_{7}=0.0331$; $\mathrm{X}_{8}=1.2513$;

O carbono estimado com o uso das funções (Tabela 2) apresentou um bom ajuste em relação àquele observado para todo o perfil (Figura 3-a e Figura 3-b). A função obtida para gleissolo apresentou um erro médio de $1,48 \mathrm{~g} \mathrm{~kg}^{-1}$ em relação aos dados observados e de $1,49 \mathrm{~g} \mathrm{~kg}^{-1}$ em relação aos dados de validação. A função para a estimação do COS em plintossolo apresentou erro médio ligeiramente superior: $1,57 \mathrm{~g} \mathrm{~kg}^{-1}$ para dados observados e 1,62 $\mathrm{g}$ $\mathrm{kg}^{-1}$ para dados de validação (Tabela 3 ).

Tabela 3 - Descritores estatísticos para o ajuste das funções, considerando dados observados e dados de validação.

\begin{tabular}{lllll}
\hline Estatísticas & $\begin{array}{l}\text { Gleissolo } \\
\text { Média }\end{array}$ & Desvio & $\begin{array}{l}\text { Plintossolo } \\
\text { Média }\end{array}$ & Desvio \\
\hline Observados & & & & \\
SSE & 97.04 & 10.92 & 179.28 & 36.85 \\
$\mathrm{R}^{2}$ & 0.88 & 0.01 & 0.77 & 0.01 \\
Adj R & 0.88 & 0.02 & 0.77 & 0.01 \\
RMSD & 1.48 & 0.08 & 1.57 & 0.17 \\
\hline Validação & & & & \\
SSE & 25.20 & 19.65 & 30.15 & 22.32 \\
RMSD & 1.49 & 0.55 & 1.62 & 0.63 \\
\hline
\end{tabular}

Fonte: elaboração dos autores.

Em relação ao intervalo de valores de concentração do COS, os piores ajustes ocorreram para maiores concentrações, superiores a $150 \mathrm{~g} \mathrm{~kg}^{-1}$ em gleissolo e a $100 \mathrm{~g} \mathrm{~kg}^{-1} \mathrm{em}$ plintossolo, conforme Figura 3 (atentar para perfis $3 \mathrm{c}$ e $3 \mathrm{~d}$ ). A partir desses valores de concentração, a função tendeu a subestimar o conteúdo de carbono no solo. 
Figura 3 - conteúdo de carbono no solo (COS) observado e estimado (a); estoque de carbonoestimado e observado (b); gráfico quantil-quantil para o COS observado e estimado em gleissolo (c); gráfico quantilquantil para o COS observado e estimado em plintossolo (d).

a)

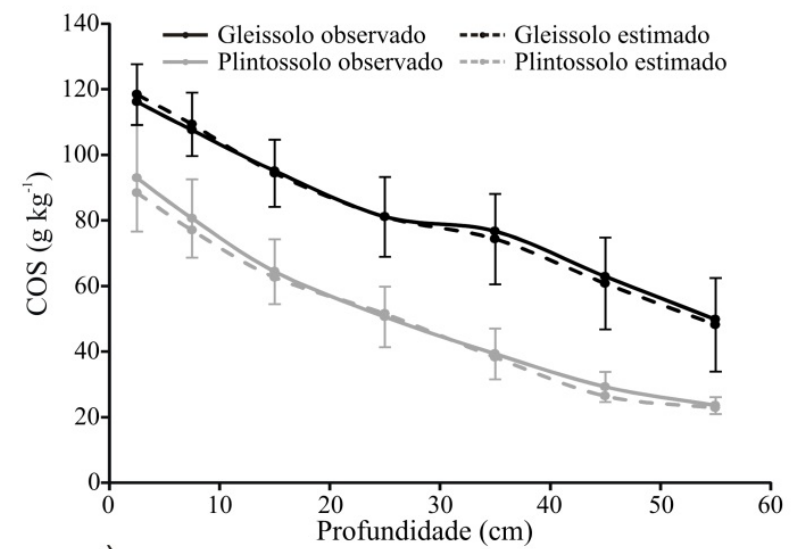

c)

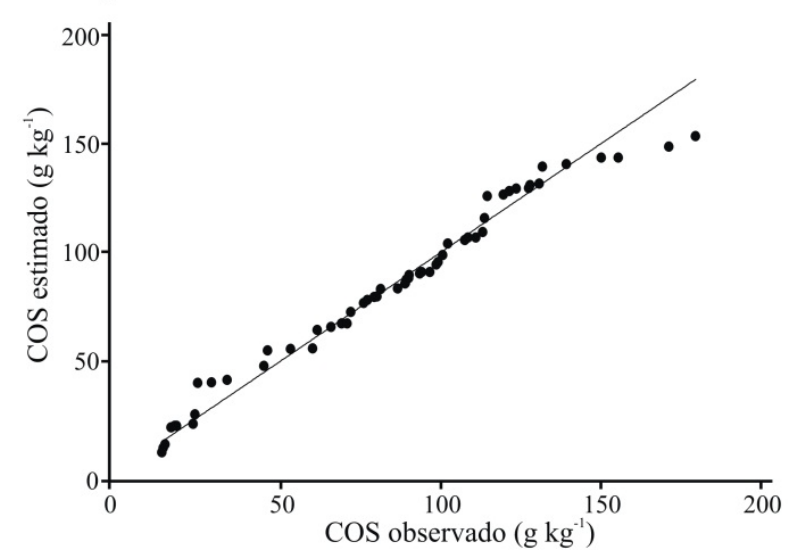

b)

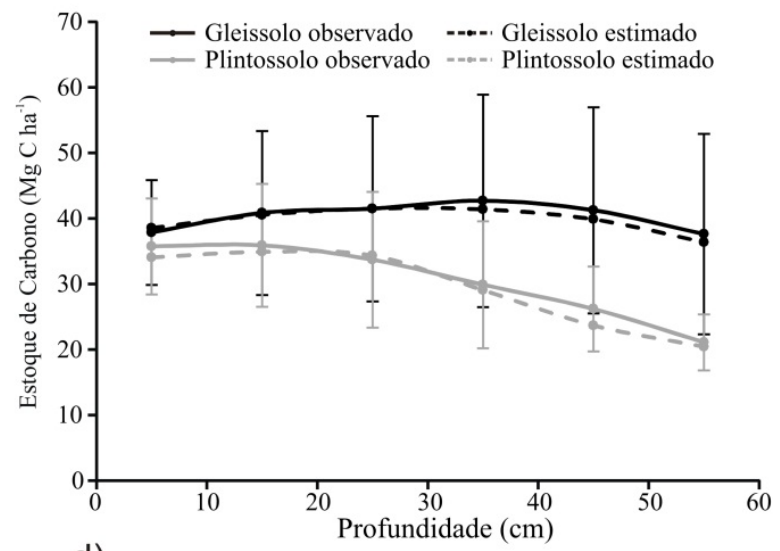

d)

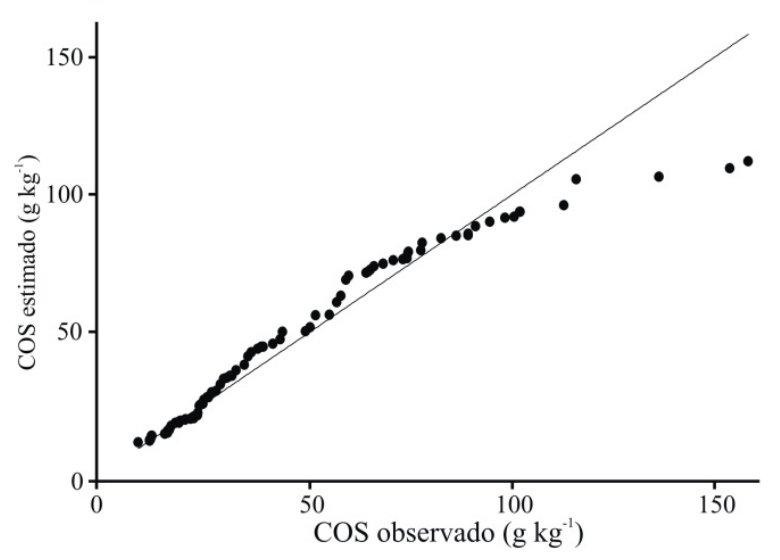

Fonte: elaboração dos autores.

\section{DISCUSSÃO}

Áreas úmidas na região tropical são reconhecidamente importantes estocadoras de carbono (BERNAL; MITSCH, 2008; BIANCHI et al., 2013; DE LA CRUZ, 1986; NEUE et al., 1997). Apesar de ainda pouco exploradas no Bioma Cerrado, os resultados obtidos neste artigo confirmam o papel desempenhado por essafitofisionomia para a manutenção docarbono orgânico no solo.

Adensidade do COS em áreas de Campo Limpo Úmido apresentou valor superior àquele observado para outras fitofisionomias típicas de áreas bem drenadas do Cerrado(Tabela 4). Em áreas de savana (cerrado e cerrado sensu stricto) o COS

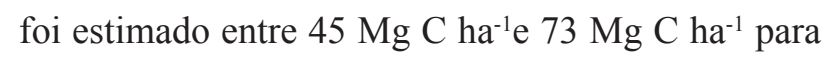
profundidade de até $60 \mathrm{~cm}$ (Tabela 4), valor até 5 vezes menor do que a densidade do estoque obtida para as áreas úmidas analisadas.Em regiões com formações campestres, com ocorrênciaemregiões bem drenadas(com solos de tipo Ferrasols), a densidade do estoque de carbono também foi bastante inferior àquelas obtidas para áreas de Campo Limpo Úmido (Tabela 4). Apesar da semelhança fitofisionômica entre essas regiões, campos limpos tiveram o estoque do solo estimado em $100 \mathrm{Mg} \mathrm{C}$ ha ${ }^{-1}$ para a profundidade de até $100 \mathrm{~cm}$ (SILVA et al., 2004), mais de duas vezes inferior àquele observado em áreas de Campo Limpo Úmido. 
Tabela 4-Estimativas da densidade dos estoques de carbono do solo em diferentes fitofisionomias do Bioma Cerrado.

\begin{tabular}{|c|c|c|c|c|c|}
\hline \multirow[b]{2}{*}{ Fitofisionomia } & \multirow{2}{*}{$\begin{array}{l}\text { Tipo de solo } \\
\text { (FAO) }\end{array}$} & \multicolumn{3}{|c|}{ Estoque (Mg C ha-1) } & \multirow[b]{2}{*}{ Referências } \\
\hline & & $0-60 \mathrm{~cm}$ & $0-100 \mathrm{~cm}$ & $0-200 \mathrm{~cm}$ & \\
\hline Cerradão & Ferralsols & - & 148 & 230 & ChapuisLardy et al. (2002) \\
\hline Cerrado & Ferralsols & - & 143 & 231 & ChapuisLardy et al. (2002) \\
\hline Cerrado & Ferralsols & - & 133 & - & Corazza et al. (1999) \\
\hline Cerrado & Ferralsols & 45 & - & - & Resck et al. (2008) \\
\hline Cerrado & Cambisols & - & 200 & - & Silva et al. (2008) \\
\hline Cerrado sensu stricto & Ferralsols & - & & 271 & Paiva e Faria (2007) \\
\hline Cerrado sensu stricto & Regosols & 50 & - & - & Zinn et al. (2002) \\
\hline Cerrado sensu stricto & Ferralsols & 74 & - & - & Zinn et al. (2002) \\
\hline Campo Sujo & Ferralsols & - & 209 & 297 & ChapuisLardy et al. (2002) \\
\hline Campo Limpo & Ferralsols & - & 198 & 281 & ChapuisLardy et al. (2002) \\
\hline Campo Limpo & Ferralsols & - & 100 & - & Silva et al. (2004) \\
\hline Campo Limpo Úmido & Histosols & 241 & - & - & Meirelles et al. (2006) \\
\hline
\end{tabular}

Fonte: Adaptado de Batlle-bayer, Batjes, \&Bindraban (2010).

De forma semelhante a outros tipos fitofisionômicos, áreas de Campo Limpo Úmido apresentaram relação entre características do solo (tipo de solo, textura, densidade) e o conteúdo de carbono orgânico nele presentes. No caso observado, as amostras obtidas em gleissolo, em geral, apresentaram conteúdo de carbono superior àquelas de plintossolo. $\mathrm{O}$ tratamento independente entre amostras pertencentes a solos com características físicas distintas, parece ser uma estratégia adequada para a análise de amostras obtidas em Campo Limpo Úmido.

Como observado em outros estudos que modelaram o COS em regiões do Bioma Cerrado (FRANÇA, 2011; REIN; DUXBURY, 2008; ZINN et al., 2012), foi possível um ajuste adequado de uma função de estimação a partir de informações menos onerosase de mais fácil obtenção (ex: densidade e textura). Esse tipo de abordagem contribui para uma maior agilidade e menor dispêndio de recursos em trabalhos que envolvam a avaliação dos estoques existentes na região de estudo.

Considerando que Campos Limpos Úmidos são áreas que se caracterizam como importantes estocadores de carbono no solo, os quais potencialmente podem ser liberados para a atmosfera em caso do manejo inadequado ou alterações ambientais que afetem o ciclo hidrológico natural, érecomendável a realização de estudos específicos que investiguem variações no $\operatorname{COS}$ decorrentes da mudança de uso e manejo em regiões de Campo Limpo Úmido, bem como a realização de pesquisas que abranjam toda a área do Bioma Cerrado.

\section{CONCLUSÕES}

Os resultados obtidos mostraram que Campos Limpos Úmidos, uma das fitofisionomias presentes no Bioma Cerrado, possuem elevado potencial para estocar carbono no solo, com uma estimativa de 
243.16 Mg Cha-1 até a profundidade $60 \mathrm{~cm}$. O estoque total estimado para essas áreas no Distrito Federal foi de 205.86Gg C, presentes em 846.61 ha de Campo Limpo Úmido. Os resultados obtidos indicaram uma diferença entre áreas sobre gleissolo e plintossolo. Em gleissolos, o estoque médio de $258.84 \mathrm{Mg} \mathrm{C}$ ha${ }^{1}$, enquanto áreas de plintossolos apresentaram em média214.49Mg C ha ${ }^{-1}$. Foram obtidas equações capazes de estimar o conteúdo de carbono orgânico no solo (COS) a partir de informações de mais fácil acesso (densidade e textura). Os valores estimados pela função apresentaram boa adequação aos dados observados em campo. A função para gleissolo apresentou um erro médio de $1,48 \mathrm{~g} \mathrm{~kg}^{-1}$ em relação aos dados observados e de $1,49 \mathrm{~g} \mathrm{~kg}^{-1} \mathrm{em}$ relação aos dados de validação. A função para a estimação do COS em plintossolo apresentou erro médio ligeiramente superior: $1,57 \mathrm{~g} \mathrm{~kg}^{-1}$ para dados observados e 1,62 $\mathrm{g} \mathrm{kg}^{-1}$ para dados de teste. Considerando a grande pressão antrópica a que estão submetidas as regiões naturais no Bioma Cerrado, é recomendável a realização de estudos específicos que investiguem variações no COS decorrentes da mudança de uso e manejo em regiões de Campo Limpo Úmido ou em função de alterações ambientais. Sugere-se, ainda, que novos estudos, com área de estudo que abranja toda a área do Bioma Cerrado, sejam realizados para esse tipo de fitofisinomia.

\section{AGRADECIMENTOS}

Os autores agradecem ao CNPq-Brasil pelo auxílio concedido para a realização da pesquisa.

\section{REFERÊNCIAS}

BATLLE-BAYER, L.; BATJES, N. H.; BINDRABAN, P. S. Changes in organic carbon stocks upon land use conversion in the Brazilian Cerrado: A review. Agriculture, Ecosystems \& Environment, v.137, n.12, p.47-58, 2010. DOI: http://dx.doi.org/10.1016/j. agee.2010.02.003

BERNAL, B.; MITSCH, W. J. A comparison of soil carbon pools and profiles in wetlands in Costa Rica and Ohio. Ecological Engineering, v.4, p.311-323, 2008.
DOI: http://dx.doi.org/10.1016/j.ecoleng.2008.09.005

BIANCHI, T. S. et al. Estuarine, Coastal and Shelf Science Historical reconstruction of mangrove expansion in the Gulf of Mexico : Linking climate change with carbon sequestration in coastal wetlands. Estuarine, Coastal and Shelf Science, v.119, p.7-16, 2013. DOI: http://dx.doi.org/10.1016/j. ecss.2012.12.007

COLETTI, J. Z. et al. Hydrological controls on carbon metabolism in wetlands. Ecological Modelling, v. 249, p. 3-18, 2013. DOI: http://dx.doi.org/10.1016/j. ecolmodel.2012.07.010

DE LA CRUZ, A. A. Tropical wetlands as a carbon source. Aquatic Botany, v.25, p.109-115, 1986. DOI: http://dx.doi.org/10.1016/0304-3770(86)90048-3

EITEN, G. The Cerrado Vegetation of Brazil. The Botanical Review, v.38, n.2, p.201-327, 1972.

EMBRAPA. Sistema Brasileiro de Classificação de Solos. Rio de Janeiro: Embrapa-SPI, 2006. p.306pp

FAO. World reference base for soil resources 2006: a framework for international classification, correlation and communication. Rome: FAO, 2006. p.1-145

FERREIRA, E. A. B. et al. Influência de variações ambientais sobre o fluxo de $\mathrm{CO} 2 \mathrm{em}$ solos sob Campo Limpo Úmido no Cerrado. II Simpósio internacional de Savanas Tropicais, v.3, p.7pp, 2008.

FRANÇA, A. M. DA S.; SANO, E. E. Influência de variações ambientais sobre o fluxo de $\mathrm{CO} 2 \mathrm{em}$ solos sob Campo Limpo Úmido no Cerrado. Sociedade e Natureza, v.2, p.197-209, 2011.

FRANÇA, A. M. DA S. Função de pedotransferência para estimativa de estoques de carbono em solo de áreas de Campo Limpo Úmido do Distrito Federal. 2011. Tese (Doutorado em Geologia) - Instituto de Geociências, Depto de Geologia, Universidade de Brasília, Brasília. 2011. 
GRACE, J. et al. Productivity andcarbonfluxesof tropical savannas. Journal of Biogeography, v.33, n.3, p 387-400, mar. 2006. DOI: http://dx.doi.org/10.1111/ j.1365-2699.2005.01448.x

IBAMA. Projeto de monitoramento do desmatamento dos biomas brasileiros por satélite PMDBBS. Disponível em: <siscom.ibama.gov.br/ monitorabiomas/cerrado/index.htm>. Acesso em: 1 maio de 2014.

LAL, R.; FOLLETT, R. F. (EDS.). Soil carbon sequestration and the greenhouse effect. Madison WI: Soil Science SocietyofAmerica, 2009. p. 410pp LEPSCH, I. F. Formação e Conservação Dos Solos. São Paulo: Ofina de Textos, 2002. 178p.

LEVENBERG, K. A Method for the Solution of Certain Non-Linear Problems in Least Squares. Quarterly of Applied Mathematics, v.2, p.164-168, 1944.

MARQUARDT, D. An Algorithm for Least-Squares Estimation of Nonlinear Parameters. SIAM Journal on Applied Mathematics, v.11, n.2, p.431-441, 1963.

MATLAB, The MathWorks Inc. MATLAB R2013a. Natick, Massachusetts, United States. 2013. Disponível em: $<$ http://www.mathworks.com/>

MEIRELLES, M. L.; FERREIRA, E. A. B.; FRANCO, A. C. Dinâmica Sazonal do Carbono em Campo Úmido do Cerrado. Documentos/Embrapa Cerrados, v.164, p 32p, 2006.

MUNHOZ, C. B. R.; FELFILI, J. M. Fitossociologia do estrato herbáceo-subarbustivo em campo limpo úmido no Brasil Central 1. Acta Botanica Brasilica, v.22, n.4, p.905-913, 2008. DOI: http://dx.doi. org/10.1590/S0102-33062008000400002

NEUE, H. U. et al. Carbon in tropical wetlands. Geoderma, v.1, p.163-185, 1997. DOI: http://dx.doi. org/10.1016/S0016-7061(97)00041-4
OLIVEIRA, J. B.; JACOMINE, P. K. T.; CAMARGO, M. N. Classes Gerais de Solos do Brasil. Guia Auxiliar para seu Reconhecimento. Jaboticabal: FUNEP, 1992. $201 \mathrm{p}$.

REATTO, A. et al. Solos de ocorrência em duas áreas sob matas de galeria no Distrito Federal: aspectos pedológicos, uma abordagem química e físico-hídrica. In: RIBEIRO, J. F.; FONSECA, C. E. L.; SOUSASILVA, J. CARLOS (Eds.). Cerrado: caracterização e recuperação de matas de galeria. Brasília: Embrapa Cerrados, 1998. p.115-140.

REIN, T. A.; DUXBURY, J. M. Modeling the soil organic carbon, texture and mineralogy relations in the profile of oxisols from the brazilian. In: SIMPÓSIO INTERNACIONAL DE SAVANAS TROPICAIS, 2., 2008, Brasília - DF. Anais do II Simpósio internacional de Savanas Tropicais, Planaltina, DF: Embrapa Cerrados, 2008.

SCURLOCK, J. M. O.; HALL, D. O. The global carbon sink : a grassland perspective. Global Change Biology, n.4, p.229-233, 1998. DOI: http://dx.doi. org/10.1046/j.1365-2486.1998.00151.x

SILVA, J. E. et al. Carbon storage in clayey Oxisol cultivated pastures in the Cerrado region, Brazil. Agriculture, Ecosystems \& Environment, v.103, p.357-363, 2004. DOI: http://dx.doi.org/10.1016/j. agee.2003.12.007

SILVA, J. F. et al. Spatial heterogeneity, land use and conservation in the cerrado region of Brazil. Journal of Biogeography, p.536-548, 2006. DOI: http://dx.doi. org/10.1111/j.1365-2699.2005.01422.x

TIESSEN, H.; MOIR, J. O. Total and organic carbon. In: CARTER, M. E. (Ed.). Soil Sampling and Methods of Analysis. Ann Arbor, Michigan: Ed. Lewis Publishers, 1993. p.187-211.

WAlter, B. M. T.; CARVAlho, A. M. DE; RIBEIRO, J. F. O Conceito de Savana e de seu Componente Cerrado. In: SANO, S. M.; ALMEIDA, S. P. DE; RIBEIRO, J. F. (Eds.). Cerrado: Ecologia e 
Flora. Brasília, DF: Embrapa Informação Tecnológica, 2008. p.19-45.

ZINN, Y. L. et al. Edaphic Controls on Soil Organic Carbon Retention in the Brazilian Cerrado: Soil Structure. Soil Science Society of America Journal, v.71, n.4,p.1204, 2007. DOI: http://dx.doi.org/10.2136/ sssaj2006.0015
ZINN, Y. L. et al. Perfis de carbono orgânico do solo nas regiões sul e Serra do Espinhaço meridional, Minas Gerais: Modelagem em profundidade. Revista Brasileira de Ciências do Solo, v.36, n.5, p.13951406, 2012. DOI: http://dx.doi.org/10.1590/S010006832012000500003 